Research Article

\title{
Solidification Microstructure Evolution of Undercooled Cu-15 wt.\% Fe Alloy Melt
}

\author{
Xiaosi Sun $\mathbb{D}$, ${ }^{1}$ Weixin Hao $\mathbb{D},{ }^{1}$ Guihong Geng $\mathbb{D}^{2},{ }^{2}$ Teng Ma $\mathbb{D},{ }^{1}$ and Yongtang $\mathrm{Li}^{1}{ }^{1}$ \\ ${ }^{1}$ School of Materials Science and Engineering, Taiyuan University of Science and Technology, Taiyuan 030024, China \\ ${ }^{2}$ School of Materials Science and Engineering, North Minzu University, Yinchuan 750000, China \\ Correspondence should be addressed to Weixin Hao; wxhao@vip.sina.com and Yongtang Li; liyongtang@tyust.edu.cn
}

Received 3 April 2018; Revised 4 June 2018; Accepted 13 June 2018; Published 26 July 2018

Academic Editor: Yee-wen Yen

Copyright (c) 2018 Xiaosi Sun et al. This is an open access article distributed under the Creative Commons Attribution License, which permits unrestricted use, distribution, and reproduction in any medium, provided the original work is properly cited.

The solidification microstructure evolution of undercooled $\mathrm{Cu}-15 \mathrm{wt} . \%$ Fe alloy melt was studied in this study by the combined method of glass fluxing and overheating. The liquidus and peritectic reaction temperatures of $\mathrm{Cu}-15 \mathrm{wt} . \% \mathrm{Fe}$ were experimentally obtained, and the obtained results were consistent with the previous studies. Based on the experimental results and related theories, the solidification process and microstructure evolution of undercooled $\mathrm{Cu}-15 \mathrm{wt} . \% \mathrm{Fe}$ alloy melt were illustrated. The conclusions provide the basis to the further study on the liquid-phase separation and application of copper-iron alloy.

\section{Introduction}

It is well known that $\mathrm{Cu}-\mathrm{Fe}$ alloy has high strength, good plasticity, giant magnetoresistance (GMR), and excellent electrical and thermal conductivity. In addition, $\mathrm{Fe}$ is characterized by the low price and abundant resources. Therefore, $\mathrm{Cu}-\mathrm{Fe}$ alloy is widely applied in aviation, automobile, communication, and electronic manufacturing [1]. However, the solidification process of $\mathrm{Cu}-\mathrm{Fe}$ alloy is different from that of conventional metals due to the great positive enthalpy between liquid $\mathrm{Cu}$ and $\mathrm{Fe}$. When the melt cools rapidly, the liquid-phase separation occurs, thus leading to solidification below the liquid metastable miscibility gap. The liquid-phase separation of $\mathrm{Cu}-\mathrm{Fe}$ alloy has been experimentally investigated at home and abroad, and the existence of liquid metastable miscibility gap has been proved $[2,3]$. The occurrence of liquid-phase separation greatly weakens the microstructure-related properties of $\mathrm{Cu}-\mathrm{Fe}$ alloy and limits its application. Therefore, it is imperative to explore the solidification process of $\mathrm{Cu}-\mathrm{Fe}$ alloy melt in the undercooled state.

Although the process and mechanisms of peritectic transformation have been extensively studied in recent decades [4-11], the solidification process of this alloy involving metastable liquid-phase separation, nucleation, and growth is not understood completely. In addition, due to the complexity of the microstructure kinetic evolution during solidification in the metastable miscibility gap, the microstructure evolution of these alloys has not been investigated quantitatively. The study aims to investigate the microstructure evolution and phase separation of $\mathrm{Cu}-15$ wt.\% $\mathrm{Fe}$ alloy melt by combining cyclic superheating with glass melting. Its position in the phase diagram [2] is shown in Figure 1.

\section{Experiment}

$\mathrm{Cu}$ and $\mathrm{Fe}$ plates were firstly machined to remove impurities from the surface, and then the machined plates were soaked in anhydrous alcohol, followed by quickly cleaning and drying to prevent surface oxidation. In order to ensure that the cooling conditions of the sample are about the same, the weight of the sample is about $15 \mathrm{~g}$, but the weight of the sample must be the same under the same composition. The raw thin metal pieces were uniformly placed at the bottom of the crucible according to a predetermined distribution ratio, and then a certain quantity of $\mathrm{B}_{2} \mathrm{O}_{3}$ glass was covered on the top surface. In order to avoid the further oxidation of the metal, after all the fine metal pieces are melted and the superheating of the melt reached $150 \mathrm{~K}$, the heat 


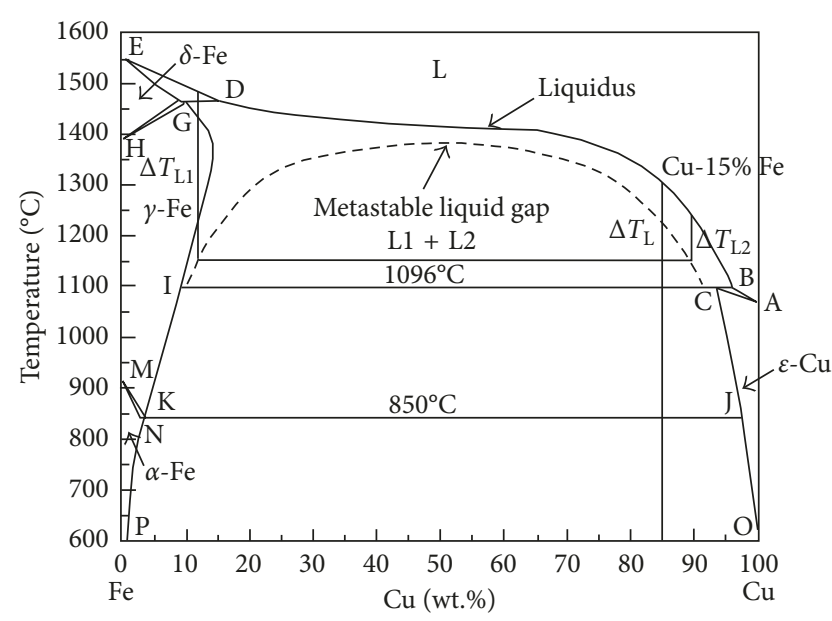

Figure 1: Phase diagram of the $\mathrm{Cu}-\mathrm{Fe}$ binary alloy with the metastable miscibility gap [2].

preservation was carried out for 3 to $5 \mathrm{~min}$, and then the sample was slowly cooled in the furnace. After complete solidification, the sample was heated again to the maximum temperature. These procedures were repeated several times until the alloy achieved the desired undercooling state. Then the sample was taken out.

The samples were embedded, polished, and corroded, and then the microstructure was analyzed by the $4 \mathrm{XA}$ HITACHI microscope and HITACHI S-3000N scanning electron microscope. The etchant was $\mathrm{FeCl}_{3}+\mathrm{HCl}+\mathrm{H}_{2} \mathrm{O}$ solution. The microstructure, phase structure, phase transition process, and composition of the samples were analyzed by the optical microscope, scanning electron microscope (SEM), and X-ray diffractometer (XRD), respectively. The phase structures of some samples were analyzed on the Rigaka D/max-RB X-ray diffractometer produced by Japanese Science Company. The scanning angle ranged from $20^{\circ}$ to $100^{\circ}$.

\section{Results and Discussion}

3.1. Solidification Process. Figure 2 illustrates the measured cooling profiles of three $\mathrm{Cu}-15$ wt. $\%$ Fe samples at different undercooling states. On the curve (a), there was a recalescence corresponding to the growth of primary $\gamma$-Fe dendrites (at about $1543 \mathrm{~K}$ ) and then led to thermal arrest, which determined the liquidus temperature, $T_{\mathrm{L}} \approx 1578 \mathrm{~K}$. The measured $T_{\mathrm{L}}$ was consistent with the value in the equilibrium phase diagram [2], thus confirming the present temperature calibration. Moreover, it is also confirmed in Figure 3, since $\gamma$-Fe dendrites are clearly visible. For all the curves in Figure 2, after further cooling, the peritectic reaction can be observed at $T_{\mathrm{P}}$ (at about $1369 \mathrm{~K}$ ). At the higher undercooling, additional anomalies appear before the major recalescence, as marked by $T_{S}$ in Figure 2(b). This unusual phenomenon neither emerged at low undercooling, nor arose from the flux agent due to the low-transition temperature (lower than $800 \mathrm{~K}$ ) of the glass. Accordingly, it can be deduced that the anomalous behavior on the curve (b) can be attributed to a precursor reaction [12], namely, a phase

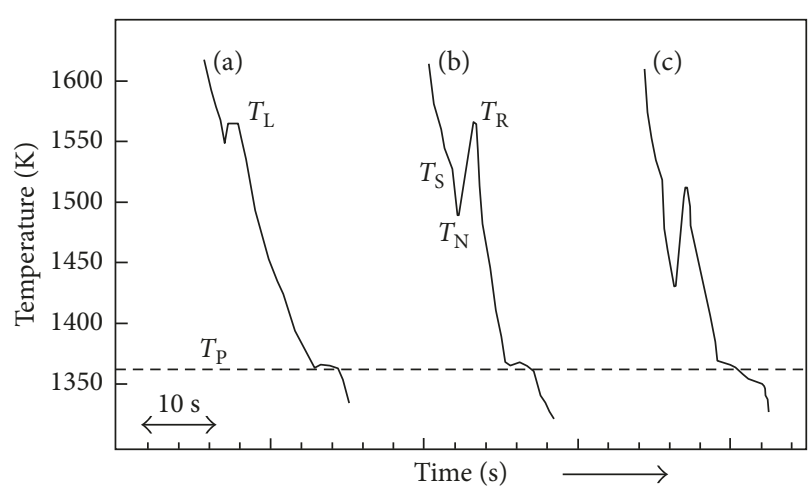

FIgURE 2: Measured cooling curves of three $\mathrm{Cu}-15$ wt.\% Fe samples: (a) $\Delta T=35 \mathrm{~K}$; (b) $\Delta T=75 \mathrm{~K}$; and (c) $\Delta T=141 \mathrm{~K} . T_{\mathrm{P}}, T_{\mathrm{N}}, T_{\mathrm{S}}$, and $T_{\mathrm{R}}$ are, respectively, peritectic, solid nucleation, liquid separation, and maximum recalescence temperatures.

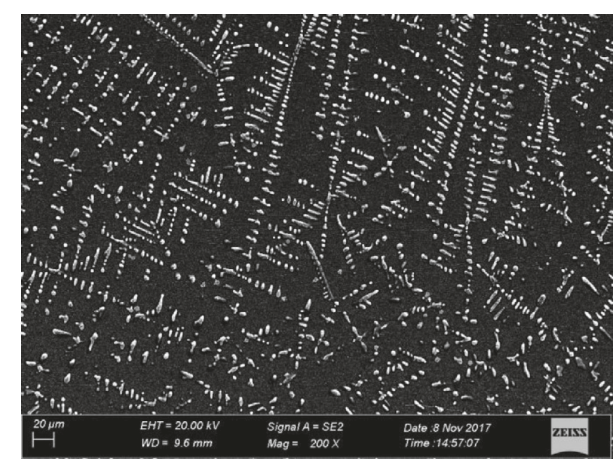

Figure 3: Microstructure of $\mathrm{Cu}-15$ wt. $\% \mathrm{Fe}$ at $\Delta T=35 \mathrm{~K}$.

separation in the sufficiently undercooled melts. The angular point on the cooling curve before the recalescence onset is hereafter referred to as $T_{S}$, the temperature at which the separation process into two liquid phases L1 (Fe-rich) and L2 (Cu-rich) begins. As the undercooling increases, liquidphase separation and recalescence are more obvious, as shown in Figure 2(c).

3.2. Microstructural Selection. Figure 4(a) shows the liquidphase separation microstructure of $\mathrm{Cu}-15$ wt.\% $\mathrm{Fe}$ at the undercooling of $75 \mathrm{~K}$. The lever rule and the metastable miscibility gap of $\mathrm{Cu}-\mathrm{Fe}$ phase diagrams (Figure 1) indicate that the $\mathrm{Cu}-15$ wt.\% Fe melt at the undercooling of $75 \mathrm{~K}$ is separated into a Fe-rich melt (L1) and a Cu-rich melt (L2). Obviously, the spheres (Figure 4(a)) are the L1 (Fe-rich) minority phase [13]. Furthermore, there are a few of $\gamma$-Fe dendrites formed due to segregation of the L2 (Cu-rich) phase [14].

After the liquid-phase separation, the L1 and L2 phases are both in the undercooled state and tend to be nucleated. In terms of classic nucleation theory [15], the critical nucleation work of forming a critical nucleus, $\Delta G^{*}$, can be given as

$$
\Delta G^{*}=\frac{16 \pi}{3} \frac{\sigma^{3}}{\Delta G_{\mathrm{v}}^{2}} f(\theta),
$$

where $\sigma$ is the interfacial energy, $\Delta G_{\mathrm{v}}$ is the Gibbs free energy difference between solid and liquid, and $f(\theta)$ is the catalytic 


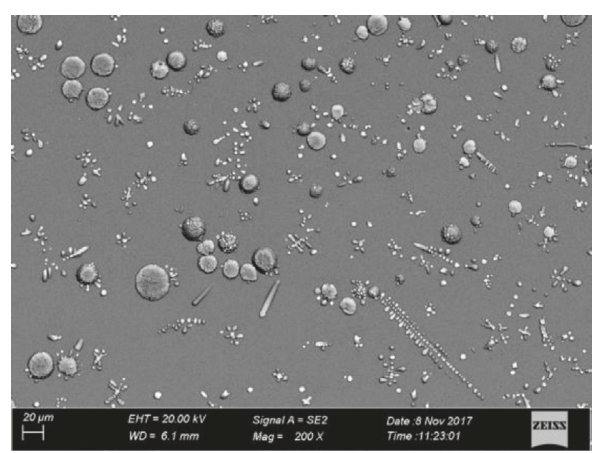

(a)

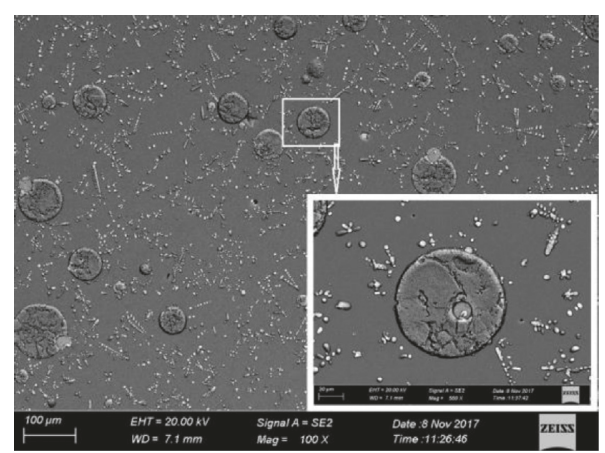

(b)

FIgURE 4: Microstructure of Cu-15 wt.\% Fe at different undercooling states: (a) $\Delta T=75 \mathrm{~K}$; (b) $\Delta T=159 \mathrm{~K}$.

factor for heterogeneous nucleation. Obviously, $\Delta G_{\mathrm{v}}$ which reflects the size of the nucleation drive follows a proportional relationship with the initial melt undercooling. As shown in Figure 1, the undercooling of the L1 (Fe-rich) phase is far higher than that of L2 (Cu-rich). As a result, $\Delta G_{\mathrm{v}}$ of the L1 (Fe-rich) phase is larger than that of the $\mathrm{L} 2(\mathrm{Cu}$-rich) phase. The L1 (Fe-rich) phase has a smaller $\Delta G^{*}$, thus resulting in a preferential nucleation for the L1 (Fe-rich) phase. Therefore, we reasonably believe that the $\mathrm{L} 1$ (Fe-rich) phase has the priority of nucleation. Furthermore, the excentric growth of L1 sphere further verifies the priority of nucleation of the L1 (Fe-rich) phase in Figure 4(a). However, in view of the thermal behavior, the solidification (forming the $\gamma$-Fe phase) of L1 and L2 is in quick succession [12]. The evidence for quick succession is shown in the curves in Figure 2. Only one major recalescence event was detected. Consequently, it is believed that the growth of one phase is prone to stimulate another.

During the further cooling process, the solute of one phase in another phase tends to be supersaturated. It should be noted that the solute is a relative concept. $\mathrm{Cu}$ in the $\mathrm{L} 1$ (Fe-rich) phase is a solute, and $\mathrm{Fe}$ in the $\mathrm{L} 2$ (Cu-rich) phase is a solute. In general, the composition of each liquid is expected to follow the miscibility phase boundary if adequate time is allowed for the complete transfer of atoms between the two phases [16]. At the same time, within a metastable miscibility gap of salient nonequilibrium features, the viscosity rises and diffusion coefficient declines with the increase in undercooling [12]. In these circumstances, complete diffusion was absent, and secondary separation (even multiphase separation) in liquid might occur. An example is shown in Figure 4(b).

3.3. Microstructural Evolution. The microstructures at different undercooling states were observed at the same magnification (Figure 5). The droplet size increases with the increase in the undercooling because the higher the undercooling is, the larger the interval $\Delta t$ from $T_{\mathrm{S}}$ to $T_{\mathrm{N}}$ is, that is, the longer coarsening time. By assuming that the largest droplet radius, $r_{\mathrm{m}}$, can be used to quantify the extent of droplets coarsening [12], a relationship between $r_{\mathrm{m}}$ and $\Delta T$ is shown in Figure 6. Corresponding to two-characteristic undercooling states, that is, $\Delta T_{\mathrm{c} 1}=103 \mathrm{~K}$ and $\Delta T_{\mathrm{c} 2}=142 \mathrm{~K}$, the increase of $r_{\mathrm{m}}$ with $\Delta T$ can be divided into three regimes: small $r_{\mathrm{m}}$ accompanying with a slow increasing rate (i.e., $d r_{\mathrm{m}} / d \Delta T$ ) for $\Delta T<\Delta T_{\mathrm{cl}}$, and medium $r_{\mathrm{m}}$ with a steep $d r_{\mathrm{m}} / d \Delta T$ for $\Delta T_{\mathrm{c} 1}<\Delta T<\Delta T_{\mathrm{c} 2}$. This result is consistent with the previous analysis result of undercooled $\mathrm{Cu}$-Co melt [12]. However, in the later stage of liquid-phase separation, there is a slight deviation between the results in this study and previous results $[2,12]$ because the maximum undercooling achieved in the study is not large enough.

The growth and coarsening of liquid globules mainly depends on Ostwald ripening, Brownian motions, Marangoni migration, and Stokes movement. At the beginning of the liquid-phase separation, the undercooling of the melt is extremely low, and the large solubility is promoted by small droplet radius [17]. Furthermore, the diffusion coefficient of $\mathrm{Fe}$ in the liquid matrix is estimated as [18]

$$
D(T)=1.057 \times 10^{-15} T^{2} .
$$

The dynamic viscosity $\eta$ can, according to Morioka's model [19], be estimated as

$$
\eta=\frac{2}{3 \pi N_{\mathrm{A}}\left(\kappa a_{0}\right)^{2}} \sqrt{\frac{M R^{*} T}{\pi}} \exp \left(\frac{E_{\mathrm{vis}}}{R^{*} T}\right),
$$

where $N_{\mathrm{A}}$ is the Avogadro number, $M$ is molar mass, $R^{*}$ is mean radius of the spheres, and $E_{\mathrm{vis}}$ is activation energy. The parameter $k$ is defined in [19]. Combining (2) and (3), the diffusion coefficient is proportional to the temperature, and the dynamic viscosity is inversely proportional to the temperature. In the early stage of liquid-phase separation, the diffusion coefficient of drops of the L1 (Fe-rich) phase in the melt is large and the dynamic viscosity is very small due to the low temperature. As a result, the above three necessary conditions for the Ostwald ripening are obtained [2]. At the same time, a free migration and coagulation process do not occur due to the short interval $\Delta t$ with low undercooling. Consequently, the coarsening of L1 droplets is mainly resulted from the Ostwald ripening at the beginning of the liquid-phase separation. With the increase in the undercooling, the diffusion coefficient of solute Fe spheres gradually decreases due to the decrease in the temperature and the viscosity gradually increases. Furthermore, the 


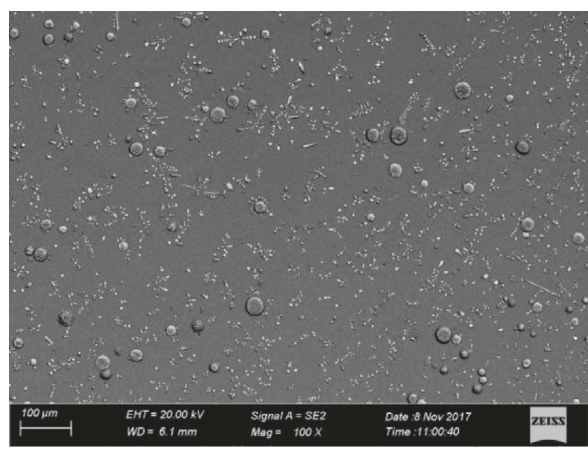

(a)

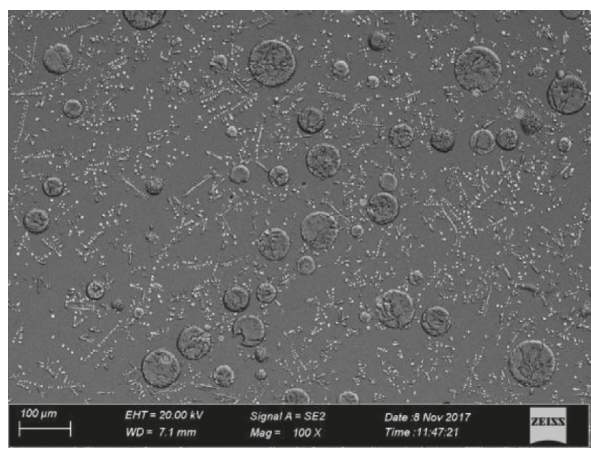

(c)

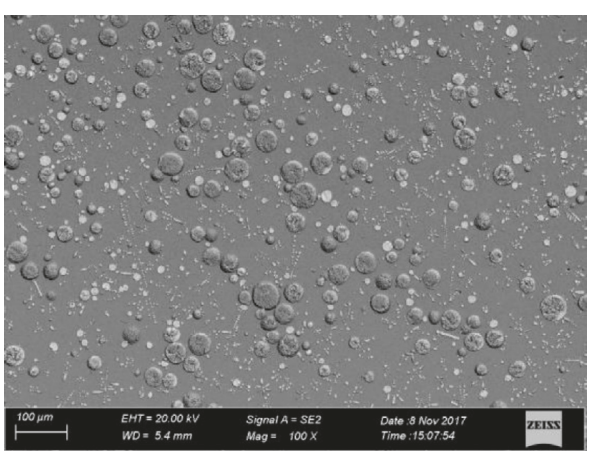

(b)

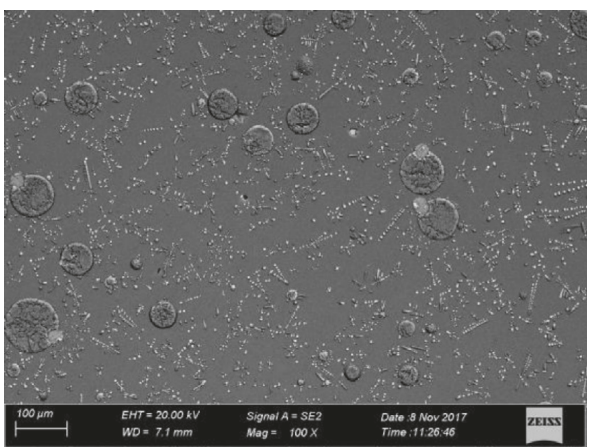

(d)

Figure 5: Microstructure of $\mathrm{Cu}-15 \mathrm{wt} . \% \mathrm{Fe}$ at different undercooling states at the same magnification of 100 times: (a) $\Delta T=75 \mathrm{~K}$; (b) $\Delta T=102 \mathrm{~K}$; (c) $\Delta T=141 \mathrm{~K}$; (d) $\Delta T=159 \mathrm{~K}$.

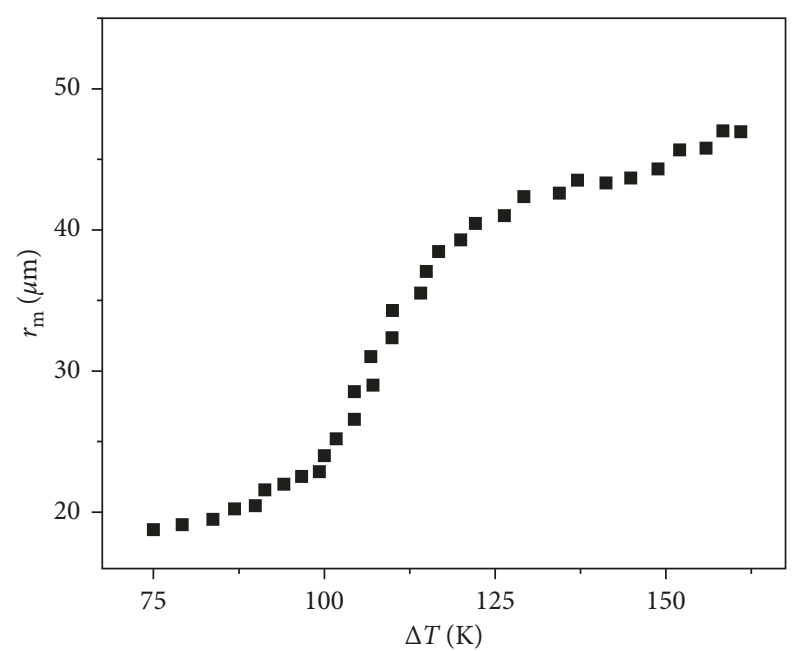

Figure 6: Relationship between the undercooling $\Delta T$ and the maximum radius $r_{\mathrm{m}}$ of Fe-rich droplets in the $\mathrm{Cu}-15 \mathrm{wt} . \% \mathrm{Fe}$ alloy.

interval $\Delta t$ is enough to allow droplet movement and coagulation, and the Brownian and Marangoni motions gradually become the dominant effect. Then, the solubility gradually decreases due to the increase in the droplet radius. Consequently, the Ostwald ripening is gradually weakened, whereas the Brownian and Marangoni motions gradually become the dominant factor of droplet coarsening. Note that the Stokes movement is still weightless due to the small size of droplets, as confirmed by (4) [20] and (5) [21]:

$$
V_{\mathrm{S}}=\frac{2}{3} \frac{\left(\rho_{2}-\rho_{1}\right)\left(\left(\eta_{2} / \eta_{1}\right)+1\right) g}{\left(3 \eta_{2}+2 \eta_{1}\right)} r^{2},
$$

where $\left(\rho_{2}-\rho_{1}\right)$ is the density difference between the solubility and matrix phases; $\eta_{1}$ and $\eta_{2}$, respectively, represent the viscosity of the matrix and the solubility phases; $g$ is the gravity acceleration vector; and $r$ is the sphere radius.

$$
V_{\mathrm{M}}=-\frac{2 k_{1} \cdot \nabla \sigma}{\left(2 k_{1}+k_{2}\right)\left(2 \eta_{1}+3 \eta_{2}\right)} r,
$$

where $k_{1}$ and $k_{2}$ are, respectively, the thermal conductivities of the matrix and solubility phases and $\nabla \sigma$ is the surface tension gradient. The main influencing factors of the Ostwald ripening are the second phase volume fraction, elastic stress, alloy elements, and so on [22], whereas the influence of the undercooling is not significant. Furthermore, the undercooling tends to inhibit the Marangoni motions, and this kind of hindrance is weak [23]. As a result, under the combined action of the Ostwald ripening, Brownian motions, and Marangoni migration, droplet size increases slowly with the increase in the undercooling at the beginning of liquid-phase separation, as confirmed in the experimental results in the study.

As the undercooling continues to increase, the size of droplets increases significantly and the effect of Stokes movement gradually becomes prominent. According to 


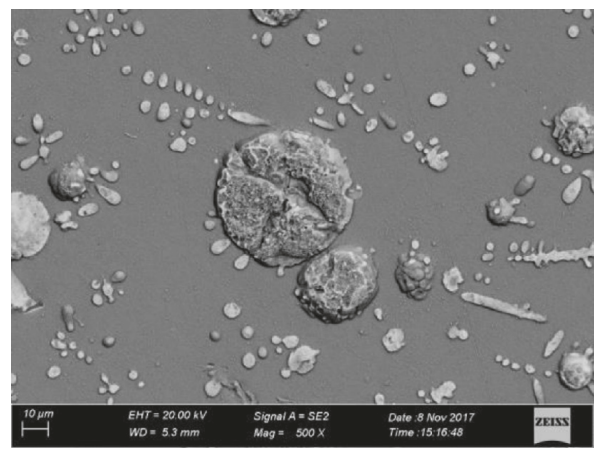

(a)

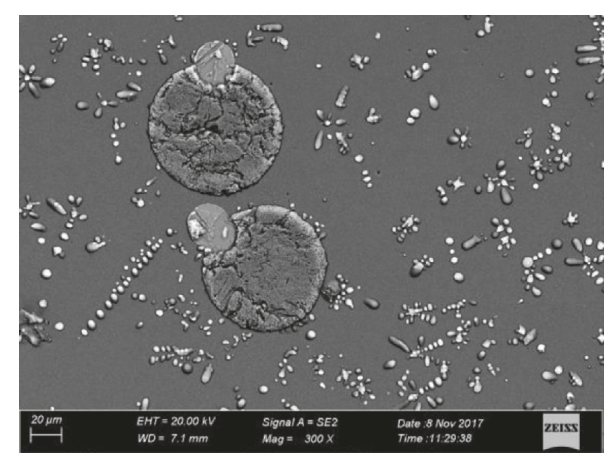

(b)

Figure 7: High-magnification microstructure of Cu-15 wt.\% Fe at different undercooling states: (a) $\Delta T=102 \mathrm{~K}$; (b) $\Delta T=159 \mathrm{~K}$.

(4) and (5) when the droplet size is larger, $V_{\mathrm{S}}$ is much larger than $V_{\mathrm{M}}$, and Ostwald ripening and Brownian motions are significantly weakened. Therefore, Stokes movement plays a dominant role in the midliquid-phase separation. Furthermore, a higher undercooling tends to facilitate Stokes movement [23]. Migration and coagulation of droplets are intensified significantly due to the gradually enhanced Stokes movement, thus resulting in the rapid increase in the droplet size. In turn, the progressively larger droplet sizes further promote Stokes movement, as shown in Figure 7. The two coagulating spheres are not in the same color because of differences in the composition between two spheres in Figure 7(b). As mentioned above, the larger sphere with the darker color is the Fe-rich sphere, and the smaller sphere with the lighter color is the $\mathrm{Cu}$-rich sphere. It is assumed that the main coagulation mechanism of the two spheres is the Ostwald ripening, but the smaller sphere with the low content of Fe coagulates with the larger sphere with the high content of Fe. The above mechanism is contrary to the Ostwald ripening mechanism due to the concentration gradient. The result confirms the above conclusion that the Stokes movement is the main mechanism. Consequently, the droplet size presents the linear growth with the increase in the undercooling in the medium stage of the liquid-phase separation. The result is consistent with that shown in Figure 6.

\section{Conclusions}

Solidification microstructure evolution of undercooled $\mathrm{Cu}$ 15 wt.\% Fe alloy melt has been described and analyzed in detail based on experimental and theoretical studies. It was found that the droplet size of undercooled $\mathrm{Cu}-15 \mathrm{wt} . \% \mathrm{Fe}$ alloy melt gradually increased with the increase in the undercooling. The increasing speed showed a slow-fastslow tendency. This tendency had been analyzed and explained theoretically. The measures for dealing with the liquid-phase separation microstructure will be explored in the future study.

\section{Data Availability}

The data used to support the findings of this study are available from the corresponding author upon request.

\section{Conflicts of Interest}

The authors declare that they have no conflicts of interest.

\section{Acknowledgments}

This work was cofinanced by the National Natural Science Foundation of China (Grant nos. 51675361 and 51561001).

\section{References}

[1] M. M. Cao, Z. M. Zhou, L. W. Tang et al., "Research progress of high strength and high conductivity Cu-Fe alloy," Materials Guide: Nano and New Material Album, vol. 25, no. 2, pp. 487-489, 2011, in Chinese.

[2] Y. Z. Chen, F. Liu, G. C. Yang et al., "Rapid solidification of bulk undercooled hypoperitectic $\mathrm{Fe}-\mathrm{Cu}$ alloy," Journal of Alloys and Compounds, vol. 427, no. 1-2, pp. L1-L5, 2007.

[3] Q. Y. Zhai, X. Zhang, J. F. Xu et al., "Rapid solidification and phase separation of cold welded foil welded joints in $\mathrm{Fe}-\mathrm{Cu}$ peritectic alloys," China Nonferrous Metals Society, vol. 19, no. 6, pp. 1080-1086, 2009, in Chinese.

[4] H. W. Kerr and W. Kurz, "Solidification of peritectic alloys," International Materials Reviews, vol. 41, no. 4, pp. 129-164, 1996.

[5] Y. Nakagawa, "Liquid immiscibility in copper-iron and copper-cobalt systems in the supercooled state," Acta Metallurgica, vol. 6, no. 11, pp. 704-711, 1958.

[6] O. Hunziker, M. Vandyoussefi, and W. Kurz, "Phase and microstructure selection in peritectic alloys close to the limit of constitutional undercooling," Acta Materialia, vol. 46, no. 18, pp. 6325-6336, 1998.

[7] K. Tokieda, H. Yasuda, and I. Ohnaka, "Formation of banded structure in $\mathrm{Pb}-\mathrm{Bi}$ peritectic alloys," Materials Science and Engineering: A, vol. 263, no. 1-2, pp. 238-245, 1999.

[8] S. Amara, A. Belhadj, R. Kersri, and S. H. Thibault, "Stable and metastable equilibria in the binary Fe-Cu and ternary Fe-Cu-C system," Zeitschrift für Metallkunde, vol. 90, no. 1948, pp. 116-123, 1999.

[9] G. Wilde and J. H. Perepezko, "Critical-point wetting at the metastable chemical binodal in undercooled $\mathrm{Fe}-\mathrm{Cu}$ alloys," Acta Materialia, vol. 47, no. 10, pp. 3009-3021, 1999.

[10] A. Munitz and R. Abbaschian, "Liquid separation in $\mathrm{Cu}-\mathrm{Co}$ and $\mathrm{Cu}-\mathrm{Co}-\mathrm{Fe}$ alloys solidified at high cooling rates," Journal of Materials Science, vol. 33, no. 14, pp. 3639-3649, 1998.

[11] I. Yamuchi, N. Ueno, M. Shimaoka, and I. Ohnaka, "Undercooling in $\mathrm{Co}-\mathrm{Cu}$ alloys and its effect on solidification 
structure," Journal of Materials Science, vol. 33, no. 2, pp. 371-378, 1998.

[12] M. B. Robinson, D. Li, T. J. Rathz, and G. Williams, "Undercooling, liquid separation and solidification of $\mathrm{Cu}-\mathrm{Co}$ alloys," Journal of Materials Science, vol. 34, no. 15, pp. 3747-3753, 1999.

[13] A. Munitz, A. Venkert, P. Landau, M. J. Kaufman, and R. Abbaschian, "Microstructure and phase selection in supercooled copper alloys exhibiting metastable liquid miscibility gaps," Journal of Materials Science, vol. 47, no. 23, pp. 7955-7970, 2012.

[14] S. Q. Liu, M. X. Yu, Y. H. Wang, W. X. Hao, and Q. S. Li, "Liquid phase separation of $\mathrm{Cu}-30 \% \mathrm{Fe}$ alloy in rapid solidification process," Chinese Journal of Rare Metals, vol. 34, no. 1, pp. 44-47, 2010.

[15] D. Turnbull, "Under what conditions can a glass be formed?," Contemporary Physics, vol. 10, no. 5, pp. 473-488, 1969.

[16] A. Munitz, A. M. Bamberger, S. Wannapanhun, and R. Abbaschian, "Effects of supercooling and cooling rate on the microstructure of $\mathrm{Cu}-\mathrm{Co}-\mathrm{Fe}$ alloys," Journal of Materials Science, vol. 41, no. 10, pp. 2749-2759, 2006.

[17] J. R. Rogers and R. H. Davis, "Modeling of collision and coalescence of droplets during microgravity processing of $\mathrm{Zn}$ Bi immiscible alloys," Metallurgical Transactions A, vol. 21, no. 1, pp. 59-68, 1990.

[18] I. Yokoyama, "Self-diffusion coefficient and its relation to properties of liquid metals: a hard-sphere description," Physica B: Condensed Matter, vol. 271, no. 1-4, pp. 230-234, 1999.

[19] S. Morioka, X. Bian, and M. Sun, "A model of viscosity for liquid metals," Zeitschrift für Metallkunde, vol. 93, no. 4, pp. 288-292, 2002.

[20] H. Lamb, Hydrodynamics, Dover, Cambridge University Press, New York, NY, USA, 6th edition, 1932.

[21] N. O. Young, J. S. Goldstein, and M. J. Block, "The motion of bubbles in a vertical temperature gradient," Journal of Fluid Mechanics, vol. 6, no. 3, pp. 350-356, 1959.

[22] Z. F. Wu and R. Wu, "Research development of secondary phase particle in dual-phase system," Materials Review, vol. 24, no. 8, pp. 113-117, 2010.

[23] X. Y. Lu, C. D. Cao, and B. B. Wei, "Microstructure evolution of undercooled iron-copper hypoperitectic alloy," Materials Science and Engineering A, vol. 313, no. 1-2, pp. 198-206, 2001. 


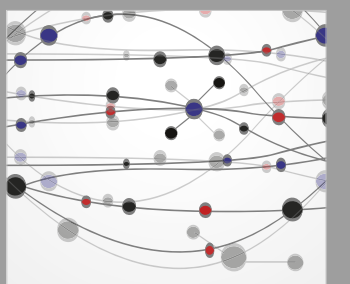

The Scientific World Journal
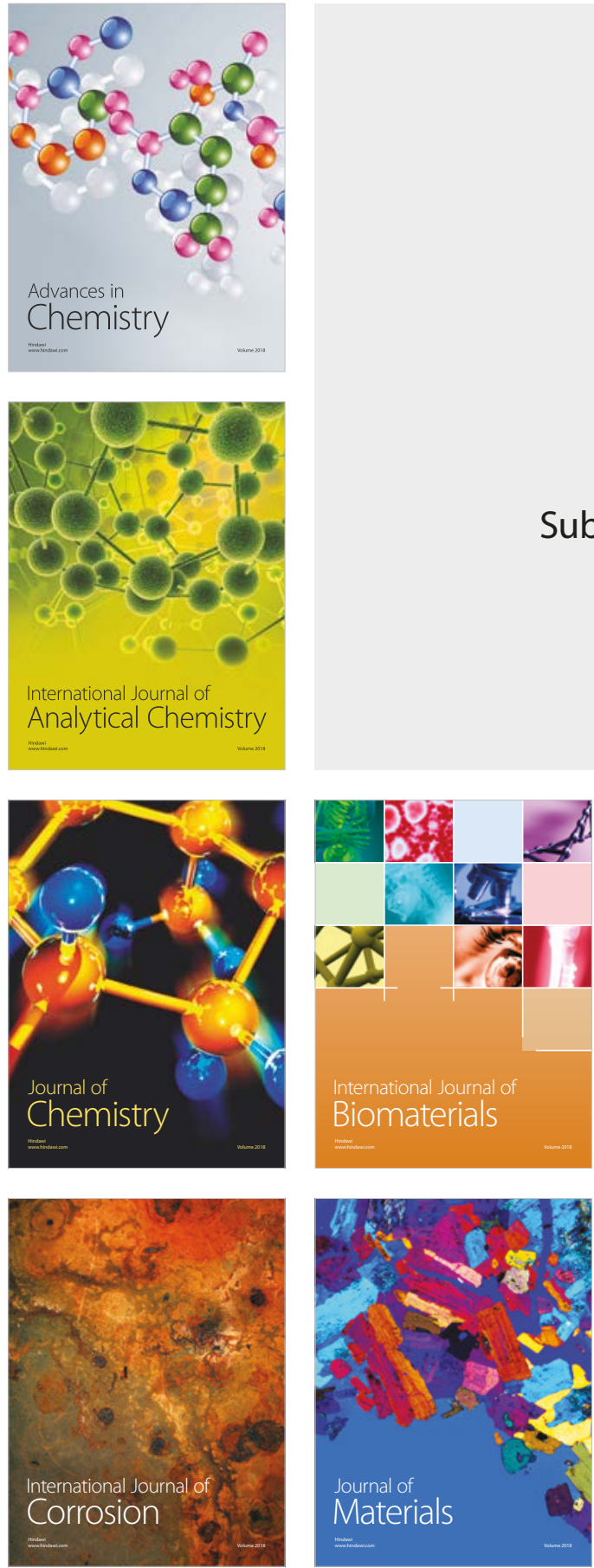

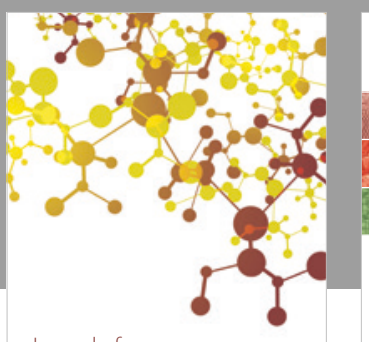

Journal of

Applied Chemistry
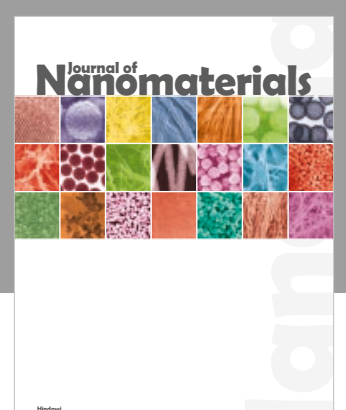

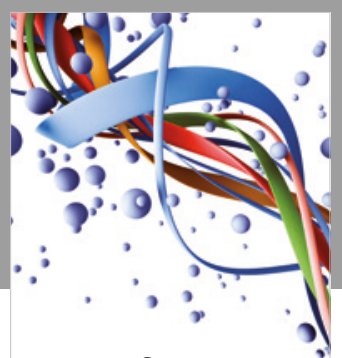

Scientifica

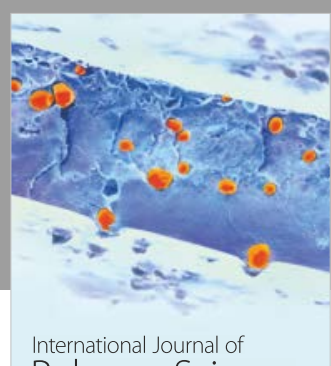

Polymer Science

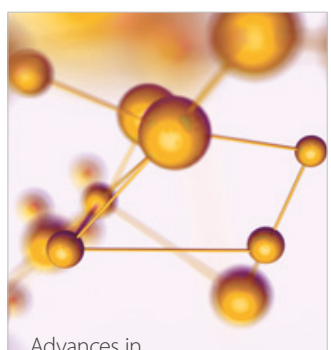

Physical Chemistry
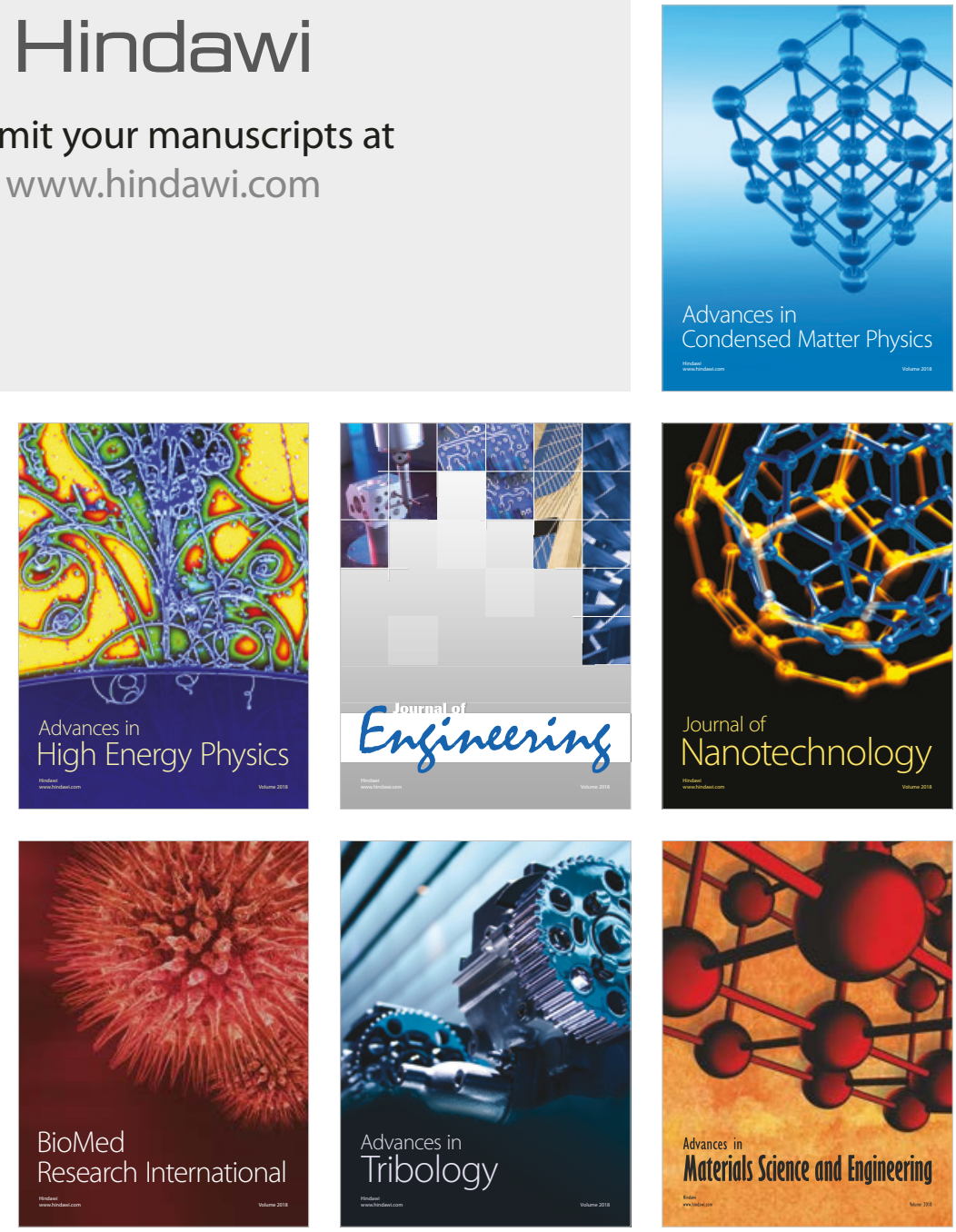